\title{
Characterization of genomic alterations and the significance of PI3K/mTOR pathway mutations and tumor mutational burden in non-small cell lung cancer
}

\author{
JING HU $^{1 *}$, YANHONG SHANG ${ }^{2 *}$, XIAOLIANG SHI ${ }^{3}$, SHUIRONG ZHANG ${ }^{3}$, JUNPING SHI $^{3}$, MING YAO $^{3}$,

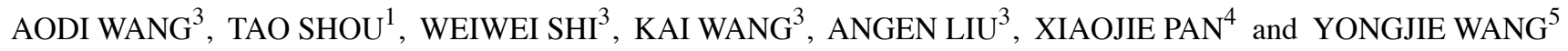 \\ ${ }^{1}$ Department of Medical Oncology, The First People's Hospital of Yunnan Province, Kunming, Yunnan 650032; \\ ${ }^{2}$ Department of Medical Oncology, Affiliated Hospital of Hebei University, Baoding, Hebei 71000; \\ ${ }^{3}$ Origimed Co., Ltd., Shanghai 201114; ${ }^{4}$ Department of Thoracic Surgery, Fujian Provincial Hospital, Fuzhou, Fujian 350001; \\ ${ }^{5}$ Department of Thoracic Surgery, The Affiliated Hospital of Qingdao University, Qingdao, Shandong 266100, P.R. China
}

Received August 23, 2019; Accepted January 23, 2020

DOI: $10.3892 /$ or.2020.7559

\begin{abstract}
Lung cancer is the most prevalent cancer worldwide and non-small cell lung cancer (NSCLC) is the most common subtype and accounts for $75 \%$ of all lung cancer cases. Although programmed death-1/programmed death-ligand-1 (PD-1/PD-L1) blockade has shown good results in the clinic, numerous NSCLC patients still fail to respond to this therapy. In the current study, formalin-fixed, paraffin-embedded tumor and matched blood samples from 1,984 Chinese NSCLS patients were collected for detection of genomic alterations including single nucleotide variations, short and long insertions/deletions, copy number variations and gene rearrangements. The most common mutated genes were tumor protein p53 $(55.70 \% ; 1,105 / 1,984)$, epidermal growth factor receptor $(52.47 \% ; 1,041 / 1,184)$, KRAS proto-oncogene GTPase $(13.36 \%, 265 / 1084)$, cyclin dependent kinase inhibitor $2 \mathrm{~A}(12.30 \% ; 244 / 1,984)$, LDL receptor
\end{abstract}

Correspondence to: Dr Yongjie Wang, Department of Thoracic Surgery, The Affiliated Hospital of Qingdao University, 59 Haier Road, Laoshan, Qingdao, Shandong 266100, P.R. China

E-mail: wangyongjie317@163.com

Dr Xiaojie Pan, Department of Thoracic Surgery, Fujian Provincial Hospital, 134 East Road, Fuzhou, Fujian 350001, P.R. China

E-mail: pxj1028@hotmail.com

Abbreviations: NSCLC, non-small cell lung cancer; PD-1/PD-L1, programmed death-1/programmed death-ligand-1; GA, genomic alteration; TMB, tumor mutational burden; TMB-H, TMB-high; TMB-L, TMB-low; FFPE, formalin-fixed, paraffin-embedded; $\mathrm{SNV}$, single nucleotide variant; $\mathrm{CNV}$, copy number variation

*Contributed equally

Key words: non-small cell lung cancer, genomic alteration, Tumor mutational burden, PI3K/mTOR pathway, programmed death-1/programmed death-ligand-1, biomarker related protein 1B $(11.09 \% ; 220 / 1,984)$ and telomerase reverse transcriptase $(10.58 \% ; 210 / 1,984)$. Tumor mutational burden was calculated and results revealed that it was associated with $\mathrm{PI} 3 \mathrm{~K} / \mathrm{mTOR}$ pathway gene mutations, and patient's gender, age, smoking status, and tumor stage. In addition, mutations of phosphatidylinositol-4,5-bisphosphate 3-kinase catalytic subunit $\alpha$ or F-box and WD repeat domain containing 7 were detected in 3 patients with NSCLC who were resistant to PD-1 inhibitors nivolumab and pembrolizumab. Disease stabilization and tumor shrinkage were observed in these patients after mTOR inhibitor everolimus treatment. The current data showed that NSCLC with PI3K/mTOR mutations are sensitive to mTOR inhibitors.

\section{Introduction}

Lung cancer is the most commonly diagnosed cancer and the leading cause of cancer-associated mortality worldwide, and non-small cell lung cancer (NSCLC) comprises $75 \%$ of all lung cancer cases (1). Immunotherapy is one of the most important treatments for $\operatorname{NSCLC}(2,3)$. The treatment involving programmed death-1/programmed death-ligand-1 (PD-1/PD-L1) blockade has produced remarkable clinical results in numerous patients with cancer and PD-1 monoclonal antibody plays an important role in the treatment of NSCLC (4-7). However, a number of cancer patients who receive PD-1/PD-L1 blockade develop resistance. The factors that contribute to immunotherapy resistance include the expression or repression of certain genes and pathways in tumor cells that prevent immune cell infiltration or function within the tumor microenvironment (8). Therefore, the development of predictive biomarkers and the identification of more effective combination therapies are still urgently needed in clinical practice for cancer management.

The PI3K/mTOR signaling pathway is associated with cell proliferation, differentiation, motility, survival and chemotherapy resistance (9-11). Alterations in the PI3K/mTOR pathway could be caused by genetic aberrations of various factors, such as phosphatidylinositol-4,5-bisphosphate 
3-kinase catalytic subunit $\alpha$ (PIK3CA) mutation and amplification, PTEN loss, and mutations of F-box and WD repeat domain containing 7 (FBXW7) and serine/threonine kinase 11, which can lead to the deregulation of the PI3K/mTOR pathway, abnormal growth, and cell proliferation (12-14). Mutations of the PI3K/mTOR pathway-related genes are frequently detected in cancer cells, and some of these genetic alterations are more prevalent in certain histological subtypes of NSCLCs (15-17).

Tumor mutational burden (TMB) is defined as the number of somatic mutations per megabase $(\mathrm{Mb})$ of the tumor coding genome (18). TMB is studied as a predictive biomarker for efficacy of immune checkpoint inhibitors treatment in solid tumors including NSCLC, melanoma and bladder cancer (8). TMB-high (TMB-H), tumors harboring a high number of gene mutations, have been reported to correlate with the generation of neoantigens and potential clinical responses to immunotherapies (19). Previous studies have found that NSCLC patients with TMB-H had higher numbers of KRAS proto-oncogene GTPase (KRAS) and tumor protein p53 (TP53) mutations than patients with low TMB (TMB-L) (20). It has previously been reported that the TMB status is correlated with the mutation of PIK3CA and FBXW7, which is involved in the PI3K/mTOR pathway in nasopharyngeal carcinoma (21). However, the relationship between the TMB status and $\mathrm{PI} 3 \mathrm{~K} / \mathrm{mTOR}$ pathway gene mutations remains unclear in NSCLC.

The current study identified the genomic alternations (GAs) in 1,984 NSCLC patients and found that mTOR pathway mutations were associated with the TMB status. These results, in combination with patients' clinical treatment data, suggested the potential of mTOR inhibitors treatment in NSCLC patients with PI3K/mTOR-related mutations.

\section{Patients and methods}

Patient enrollment and sample collection. A total of 1,984 Chinese patients with NSCLC, including 1,060 males and 924 females, were enrolled in the current study. Informed consent was obtained from all patients and this study was approved by Ethics Committees of The First People's Hospital of Yunnan Province, Affiliated Hospital of Hebei University, Fujian Provincial Hospital and The Affiliated Hospital of Qingdao University. Formalin-fixed, paraffin-embedded (FFPE) tumor tissues and matched blood samples were collected from patients and sent to OrigiMed Inc. for genetic variation detection as described below. Genomic DNA was prepared using QIAamp DNA FFPE Tissue kit and QIAamp DNA Blood Midi kit (Qiagen $\mathrm{GmbH}$ ) according to the manufacturer's instructions. The concentration of DNA was measured by a Qubit fluorometer and normalized to $20-50 \mathrm{ng} / \mu \mathrm{l}$.

Detection of serum tumor markers. Serum CA19-9, CEA, SCC, CYFRA21-1 and CA 242 levels were detected by Cobas ${ }^{\circledR}$ analyser (Roche Diagnostics) in a patient with lung squamous carcinoma who was subjected to pembrolizumab treatment. The following regimen was used: $150 \mathrm{mg}$ for two cycles, $200 \mathrm{mg}$ for the third cycle, $100 \mathrm{mg}$ for the fourth and fifth cycle, and $150 \mathrm{mg}$ for the sixth and seventh cycle. The patient was followed up for 6 months.
Detection of GAs. The genomic alterations profile was produced using the YuanSu450 ${ }^{\mathrm{TM}}$ gene panel (OrigiMed Inc.). This panel covers all the coding exons of 450 cancer-related genes and 64 selected introns in 39 genes that are frequently rearranged in solid tumors. The genes were captured and sequenced with a mean depth of $800 \mathrm{X}$ using an Illumina NextSeq 500 (Illumina, Inc.). Single nucleotide variants (SNVs) were identified by MuTect (version 1.7) method (22). Insertion-deletion polymorphisms (Indels) were identified using PINDEL (version 0.2.5) method (23). Indels $<50$ bp were considered ShortIndels, while those $>50$ bp were considered LongIndel. The functional impact of each GA was annotated by SnpEff 3.0 (24). Copy number variation (CNV) regions were identified by Control-FREEC tool (version 9.7) with the following parameters: Window $=50,000$ and step $=10,000$ (25). Gene fusions were detected through an in-house developed pipeline, as previously described (26). Gene rearrangements were assessed by Integrative Genomics Viewer (27).

$T M B$. TMB was calculated by counting the somatic mutations, including SNVs and Indels, per $\mathrm{Mb}$ of the sequence examined in each patient. Driver mutations and known germline alterations were excluded. The TMB value was further divided into two groups: TMB-H, defined as $\geq 10$ mutations $/ \mathrm{Mb}$, and TMB-L, defined as $<10$ mutations/Mb.

Statistical analysis. The categorical variables were analyzed by Fisher's exact test. The comparisons between normally distributed data and non-normally distributed data were performed using Student's t-test and Wilcoxon rank test, respectively. Associations between multiple groups of non-parametric data were analyzed by using Kruskal-Wallis test. All statistical analyses were performed with SPSS software (version 22.0; IBM Corp.). Data are presented as the median and interquartile range.

\section{Results}

Clinical characteristics of patients with NSCLC. A total of 1,984 Chinese NSCLC patients were enrolled in the current study, including 1,060 males and 924 females, with a median age of 59 year (range, 25-92 years). Tumor samples included $1,748(88.10 \%)$ adenocarcinoma and $236(11.90 \%)$ squamous cell carcinoma, of which $692(34.88 \%)$ patients with detailed pathological examination records were divided into T1-T4 stages based on the 8th edition Classification for lung cancer (28). A total of 127 patients had tumor stage I, 82 tumor stage II, 128 tumor stage III, and 355 tumor stage IV. Among 1,511 patients with smoking information, 494 had a history of smoking $>10$ years, 5 had a history of smoking $>1$ year, and 1,012 never smoked. Clinical or pathological information for each patient was obtained and is shown in Table I.

GAs in NSCLC. A total of 13,672 clinically relevant GAs were identified using next-generation sequencing targeting 450 cancer genes, with a mean of 6.89 alterations/sample (range 1-46) in 462 genes (Fig. 1; Table SI). Of the 13,672 GAs, 9,402 (68.77\%) were SNV/ShortIndel, 3,546 (25.94\%) were CNV, 420 (3.07\%) were fusion, and 304 (2.22\%) were LongIndel (Fig. 1, Table SI). The most frequent GAs were 
Table I. Clinicopathological features of the Chinese non-small cell lung cancer cohort enrolled in the current study.

\begin{tabular}{lc}
\hline Characteristic & Value \\
\hline Gender & \\
Male & $1,060(53.43 \%)$ \\
Female & $924(46.57 \%)$ \\
Age, median (range) & $59(25-92)$ \\
TMB, median (range) & $7.6(0-94.4)$ \\
Smoking status, n (\%) & \\
Smoking & $499(25.15)$ \\
Nonsmoking & $1,012(51.01)$ \\
Unknown & $473(23.84)$ \\
Histology, n (\%) & \\
Adenocarcinoma & $1,748(88.10)$ \\
Squamous cell carcinoma & $236(11.90)$ \\
Tumor stage, $\mathrm{n}$ & \\
Stage I & 127 \\
Stage II & 82 \\
Stage III & 128 \\
Stage IV & 355 \\
Unknown & 1,293 \\
\hline
\end{tabular}

TMB, tumor mutational burden.

found in TP53 $(55.70 \% ; 1,105 / 1,984)$, epidermal growth factor receptor (EGFR; 52.47\%; 1,041/1,184), KRAS (13.36\%; $265 / 1,084)$, cyclin dependent kinase inhibitor 2A (CDKN2A; $12.30 \% ; 244 / 1,984)$, LDL receptor related protein 1B (LRP1B; $11.09 \% ; 220 / 1,984)$, and telomerase reverse transcriptase (TERT; 10.58\%; 210/1,984). All other gene mutations were detected at $<10 \%$ (Table SI). Most of the genes were mutated once, while a few genes, including TP53, succinate dehydrogenase complex flavoprotein subunit A (SDHA), SET domain containing 2 histone lysine methyltransferase (SETD2), RB transcriptional corepressor 1, RELA proto-oncogene, NF-kB subunit, ROS proto-oncogene 1 receptor tyrosine kinase (ROS1), SRY-box transcription factor 2 , spectrin $\alpha$ erythrocytic 1, serine/threonine kinase 11 (STK11), TEK receptor tyrosine kinase, tyrosine kinase non receptor 2 and zinc finger protein 217, had 2 or 3 mutations in a case. Among them, TP53, SDHA and SETD2 with multiple mutation sites were detected in 2 cases of NSCLC.

Smoking history is associated with lung squamous cell carcinoma. The proportion of male patients who had smoking history was significantly higher than that of female patients (60.63 vs. $2.37 \%$; $\left.\mathrm{P}<2.2 \times 10^{-16}\right)$. Furthermore, the occurrence of lung squamous cell carcinoma was significantly associated with male gender $\left(\mathrm{P}<2.2 \times 10^{-16}\right)$. A significantly association between smoking history and lung squamous cell carcinoma was also detected $\left(\mathrm{P}<2.2 \times 10^{-16}\right)$ (Data not shown).

Association between TMB and gender, age, tumor stage and smoking history in NSCLC. TMB is an emerging genomic
Table II. Number and proportion of TMB-H/TMB-L patients in different age groups.

Number of patients

Age range TMB-high TMB-low Total TMB-H/Total (\%)

\begin{tabular}{rrrrc}
\hline $25-40$ & 2 & 99 & 101 & 1.98 \\
$41-50$ & 61 & 220 & 281 & $21.71^{\mathrm{a}}$ \\
$51-60$ & 123 & 494 & 617 & $19.94^{\mathrm{a}}$ \\
$61-70$ & 189 & 541 & 730 & $25.89^{\mathrm{a}, \mathrm{b}}$ \\
$71-80$ & 65 & 162 & 227 & $28.63^{\mathrm{a}, \mathrm{b}}$ \\
$81-92$ & 11 & 17 & 28 & $39.29^{\mathrm{a}, \mathrm{b}}$ \\
\hline
\end{tabular}

TMB, tumor mutational burden; TMB-H, TMB $\geq 10$ muts $/ \mathrm{Mb}$; TMB-L, TMB $<10$ muts/Mb. ${ }^{\mathrm{a}} \mathrm{P}<0.05$ vs. $25-40$ age range; ${ }^{\text {}} \mathrm{P}<0.05$ vs. 51-60 age range.

biomarker that measures the number of gene mutations in the tumor genome (8). To explore the relationship between TMB and clinically relevant GAs, the current study measured the TMB in all samples. The median TMB of 1,984 samples was 7.6 mutations/Mb (range, 0-94.4 mutations/Mb; Table I). The current study further analyses the relationship between TMB and gender, age, and tumor stage in NSCLC. Among males, 376 patients were identified with TMB-H and 684 patients with TMB-L. Among females, 94 patients were identified with TMB-H and 830 patients with TMB-L. The TMB-H rate of females was significantly lower compared with males $(10.17$ vs. $35.47 \%$, respectively; $\mathrm{P}<2.2 \times 10^{-16}$; Fig. $2 \mathrm{~A}$ ). All patients were divided into six groups based on age: $25-40$ years old (101 patients), 41-50 years old (281 patients), 51-60 years old (617 patients), 61-70 years old (730 patients), 71-80 years old (227 patients), and $81-92$ years old ( 28 patients). The highest TMB-H rate was $39.29 \%$ in $81-92$ years-old patients, while the lowest TMB-H rate was $1.98 \%$ in $25-40$ years-old patients (Table II). Compared with patients aged 61-92 years, a significantly lower TMB-H rate was detected in both 25-40 years old patients and 51-60 years old patients (Fig. 2B; Table II). An association was also identified between TMB and tumor stage in 692 NSCLC patients. The stage I tumors had a lower TMB compared with other tumor stages (Fig. 2C). The current study further investigated the relationship between smoking history and TMB. The results showed significant higher TMB values in smoking patients than that in nonsmoking patients (62.61 vs. $24.01 \%$; $\mathrm{P}<2.2 \times 10^{-16}$; Fig. 2D).

Association between gene mutations in the PI3K/mTOR pathway and TMB values in NSCLC. Among the most frequently mutated genes in the current study, patients with TP53, KRAS, CDKN2A and LRP1B mutations had a higher TMB value than patients without those gene mutations, whereas patients with EGFR mutations had a lower TMB value than patients without EGFR mutations (Fig. 3). The mutations of 17 genes in the PI3K/mTOR pathway, including phosphatidylinositol-4,5-bisphosphate 3-kinase catalytic subunit $\beta$ (PIK3CB), inositol polyphosphate-4-phosphatase type II B (INPP4B), PIK3R2, regulatory associated protein of 
Table III. Mutational frequency of the PI3K/mTOR pathway genes and the corresponding TMB distribution.

\begin{tabular}{|c|c|c|c|c|c|}
\hline Gene & Mutation number & $\begin{array}{c}\text { Mutation frequency } \\
(\%)\end{array}$ & TMB-H number & TMB-L number & $\begin{array}{c}\text { TMB-H rate } \\
(\%)\end{array}$ \\
\hline AKT1 & 15 & 0.76 & 2 & 13 & 13.33 \\
\hline AKT2 & 47 & 2.37 & 18 & 29 & 38.30 \\
\hline AKT3 & 6 & 0.30 & 3 & 3 & 50.00 \\
\hline FBXW7 & 30 & 1.51 & 11 & 19 & 36.67 \\
\hline INPP4B & 25 & 1.26 & 18 & 7 & 72.00 \\
\hline MTOR & 21 & 1.06 & 12 & 9 & 57.14 \\
\hline PIK3CA & 198 & 9.98 & 87 & 111 & 43.94 \\
\hline PIK3CB & 16 & 0.81 & 12 & 4 & 75.00 \\
\hline PIK3R 1 & 26 & 1.31 & 11 & 15 & 42.31 \\
\hline PIK3R2 & 10 & 0.50 & 6 & 4 & 60.00 \\
\hline PPP2R1A & 18 & 0.91 & 8 & 10 & 44.44 \\
\hline PTEN & 91 & 4.59 & 26 & 65 & 28.57 \\
\hline RICTOR & 41 & 2.07 & 14 & 27 & 34.15 \\
\hline RPTOR & 19 & 0.96 & 11 & 8 & 57.89 \\
\hline STK11 & 92 & 4.64 & 44 & 48 & 47.83 \\
\hline TSC1 & 19 & 0.91 & 4 & 15 & 21.05 \\
\hline TSC2 & 33 & 1.66 & 14 & 19 & 42.42 \\
\hline
\end{tabular}

TMB, tumor mutational burden; TMB-H, TMB $\geq 10$ muts/Mb; TMB-L, TMB $<10$ muts/Mb.

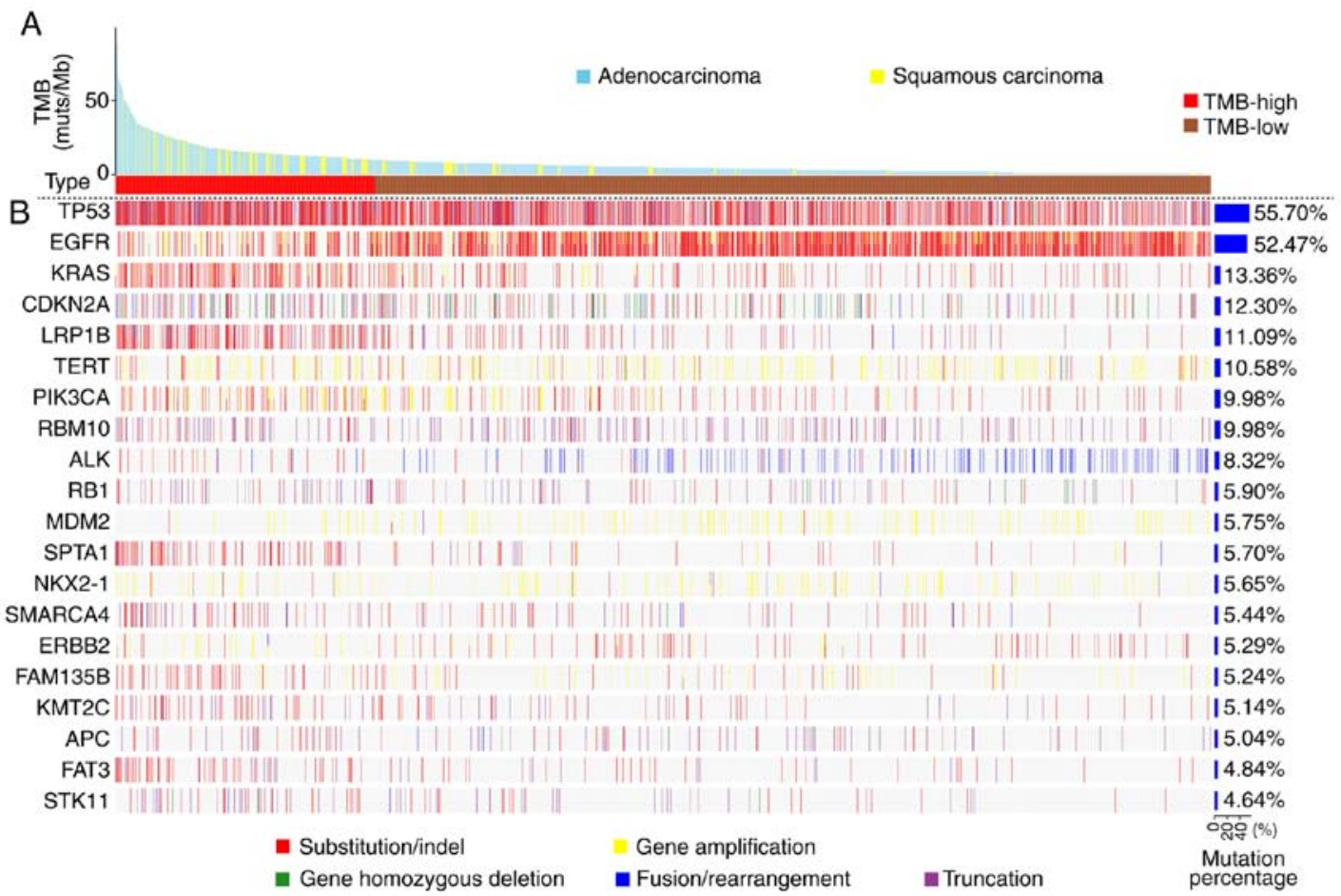

Figure 1. Most common genomic alterations in the Chinese NSCLC patients enrolled in the current study. (A) TMB (muts/Mb) in adenocarcinoma was indicated in blue and squamous cell carcinoma in yellow. The TMB values were divided into two groups: TMB-H with $\geq 10$ muts/Mb, denoted in red, and TMB-L $<10$ muts/Mb, denoted in brown. (B) Mutational landscape of Chinese NSCLC patients. The X-axis represents each case sample and the Y-axis represents each mutated gene. The bar graph on the right shows the mutation frequency of each mutated gene in 1,984 samples. Red represents substitution/Indel mutations, yellow represents gene amplification mutations, green represents gene homozygous deletion mutations, blue represents fusion/rearrangement mutations, and purple represents truncation mutations. Indel, insertion-deletion; NSCLC, non-small cell lung cancer; TMB, tumor mutational burden.

MTOR complex 1 (RPTOR), MTOR, AKT3, STK11, PIK3CA, TSC complex subunit 2 (TSC2), phosphoinositide-3-kinase regulatory subunit 1 (PIK3R1), AKT2, RPTOR independent companion of MTOR complex 2 (RICTOR), PTEN, protein 

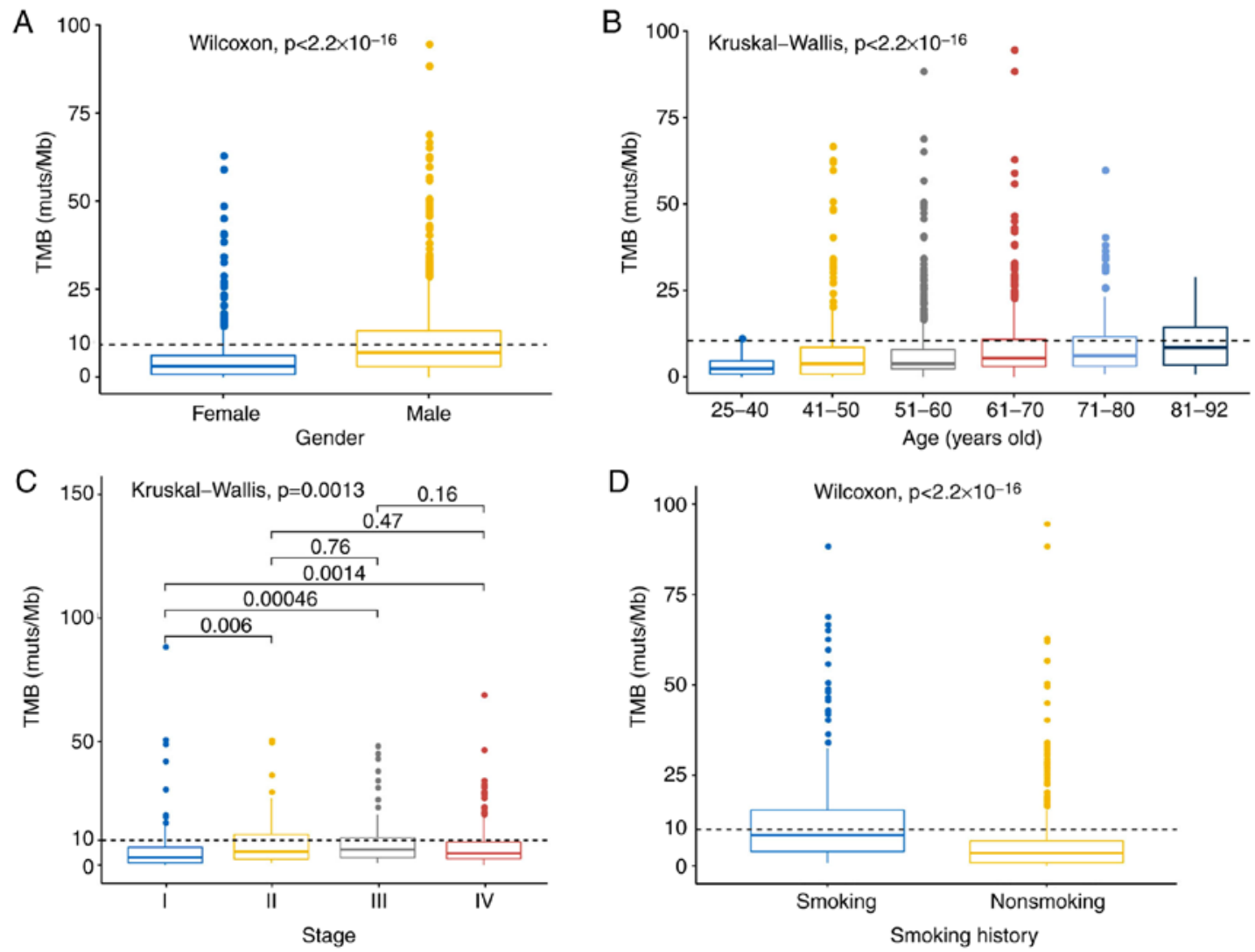

Figure 2. Association between TMB values and gender, age, tumor stage and smoking status. (A) The TMB value in females was significant lower than that in males. (B) The TMB value was significantly associated with the age of patients. (C) The TMB value was significantly higher in stage I tumors compared with stage II, III, and IV tumors. (D) The TMB value was significantly higher in smoking patients than nonsmoking patients. TMB, tumor mutational burden; Muts, mutations.
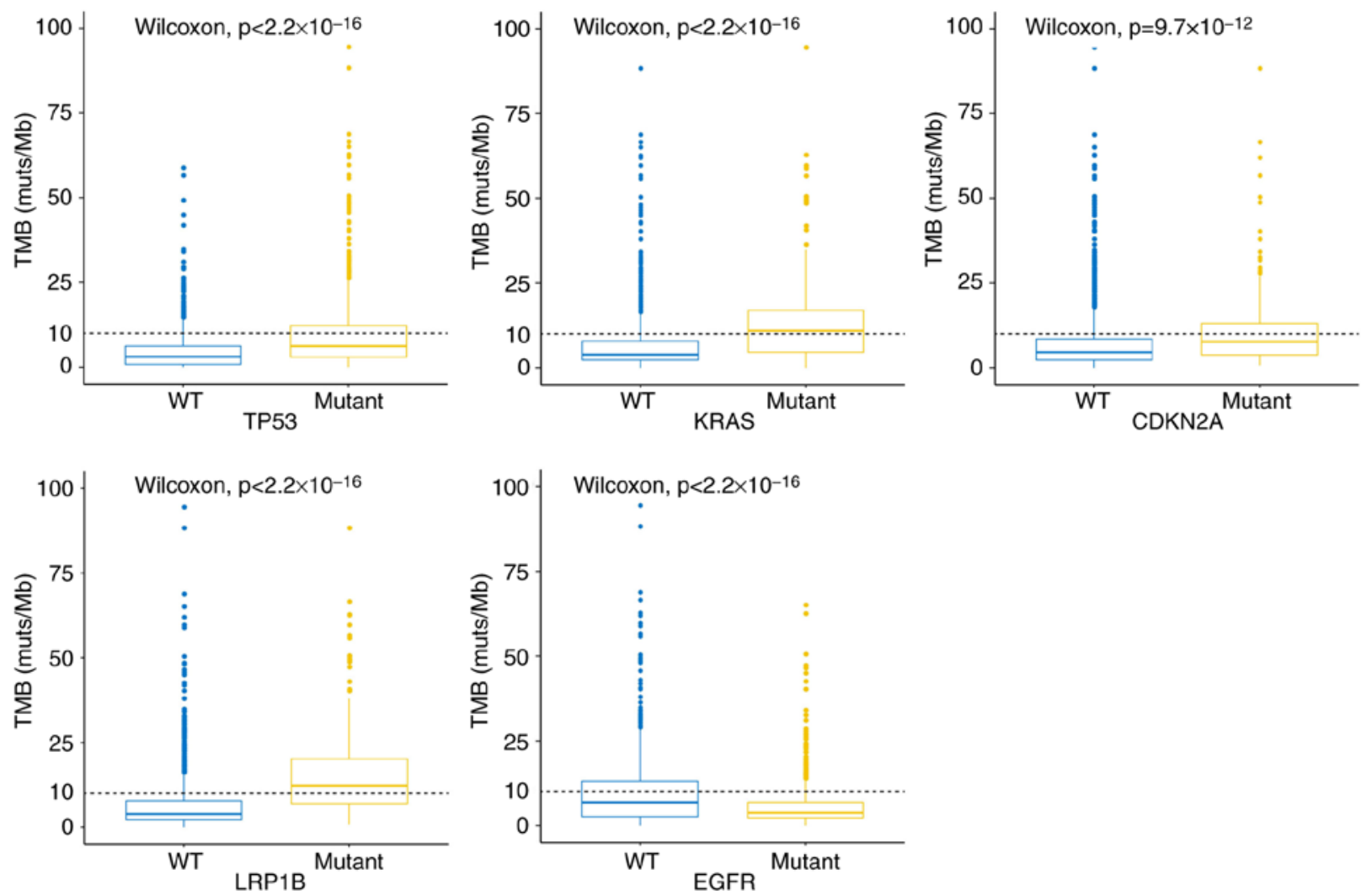

Figure 3. Association between TMB values and the most common mutated genes in NSCLC. TP53, KRAS, CDKN2A, and LRP1B mutations were more common in TMB-H patients, whereas EGFR mutations were less observed. 

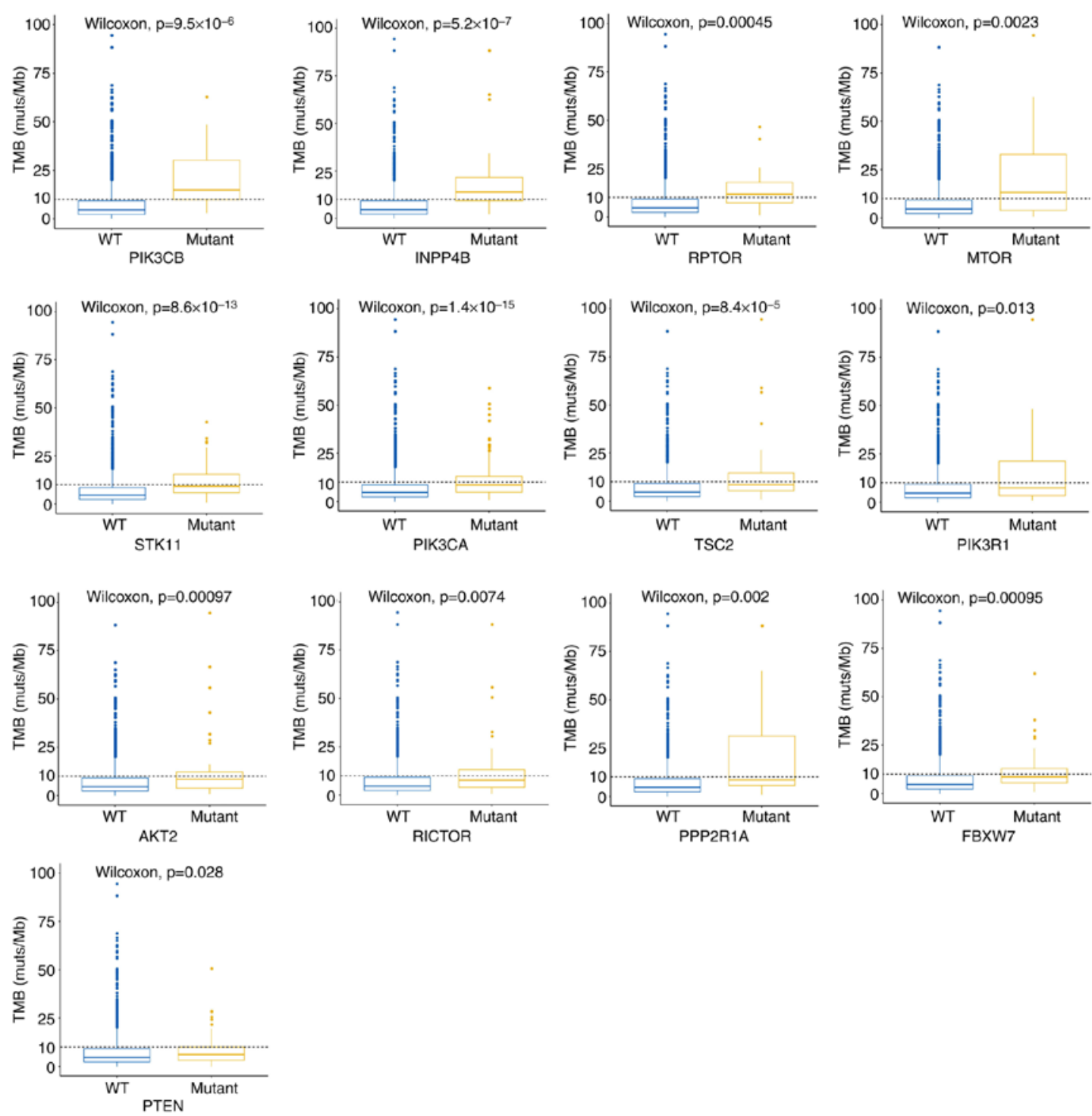

Figure 4. Association between TMB values and the mutated genes in the PI3K pathway. The mutations of PIK3CB, INPP4B, RPTOR, MTOR, STK11, PIK3CA, TSC2, PIK3R1, AKT2, RICTOR, PPP2R1A, FBXW7, and PTEN were associated with the TMB value. PIK3CB, phosphatidylinositol-4,5-bisphosphate 3-kinase catalytic subunit $\beta$; INPP4B, inositol polyphosphate-4-phosphatase type II B; RPTOR, regulatory associated protein of MTOR complex 1; STK11, serine/threonine kinase 11; PIK3CA, phosphatidylinositol-4,5-bisphosphate 3-kinase catalytic subunit $\alpha$; TSC2, TSC complex subunit 2; PIK3R1, phosphoinositide-3-kinase regulatory subunit 1; RICTOR, RPTOR independent companion of MTOR complex 2; PPP2R1A, protein phosphatase 2 scaffold subunit A $\alpha$; FBXW7, F-box and WD repeat domain containing 7.

phosphatase 2 scaffold subunit A $\alpha$ (PPP2R1A), FBXW7, TSC1, and AKT1, were also detected in this study. Except for the mutations of AKT1, TSC1 and PTEN, the mutations of most PI3K/mTOR pathway genes occurred at a high TMB-H rate ( $>50 \%$; Table III). The mutations of PIK3CB, INPP4B, RPTOR, MTOR, STK11, PIK3CA, TSC2, PIK3R1, AKT2, RICTOR, PPP2R1A, FBXW7, and PTEN were associated with the TMB value (Fig. 4).

Mutations of the PI3K/mTOR pathway in NSCLC patients with anti-PD-1 immune therapies. Among the patients included in the current study, 3 NSCLC patients, including 1 with lung adenocarcinoma and 2 with lung squamous carcinoma, had progressed after receiving $\geq 6$ cycles of anti-PD- 1 immunotherapies with pembrolizumab or nivolumab. All 3 patients failed to respond of anti-PD-1 immunotherapies. GA analysis revealed that eight clinical mutations of four genes were detected in the patient with lung adenocarcinoma, five of which occurred in the PIK3CA gene (Fig. 5A). A clinical mutation in PIK3CA and eight clinical mutations, including a mutation in FBXW7, were also detected in 2 patients with lung squamous cell carcinoma (Fig. 5A). Antigen tracking was performed on lung squamous carcinoma patient 2 who was treated with pembrolizumab. The results showed that detected antigens were downregulated with 3 cycles of treatment, but began to rebound in the following cycles, indicating PD-1 blockade resistance had occurred (Fig. 5B). Together, these results revealed that all 3 patients harbored clinically relevant gene alterations in the PI3K/mTOR pathway. Of the 3 patients, 1 maintained stable clinical benefit from mTOR inhibitor everolimus for 6 months, while the other 2 patients received mTOR inhibitor everolimus monotherapy after developing resistance to nivolumab and pembrolizumab, and both cases showed stable disease or minor shrinkage of tumors. 

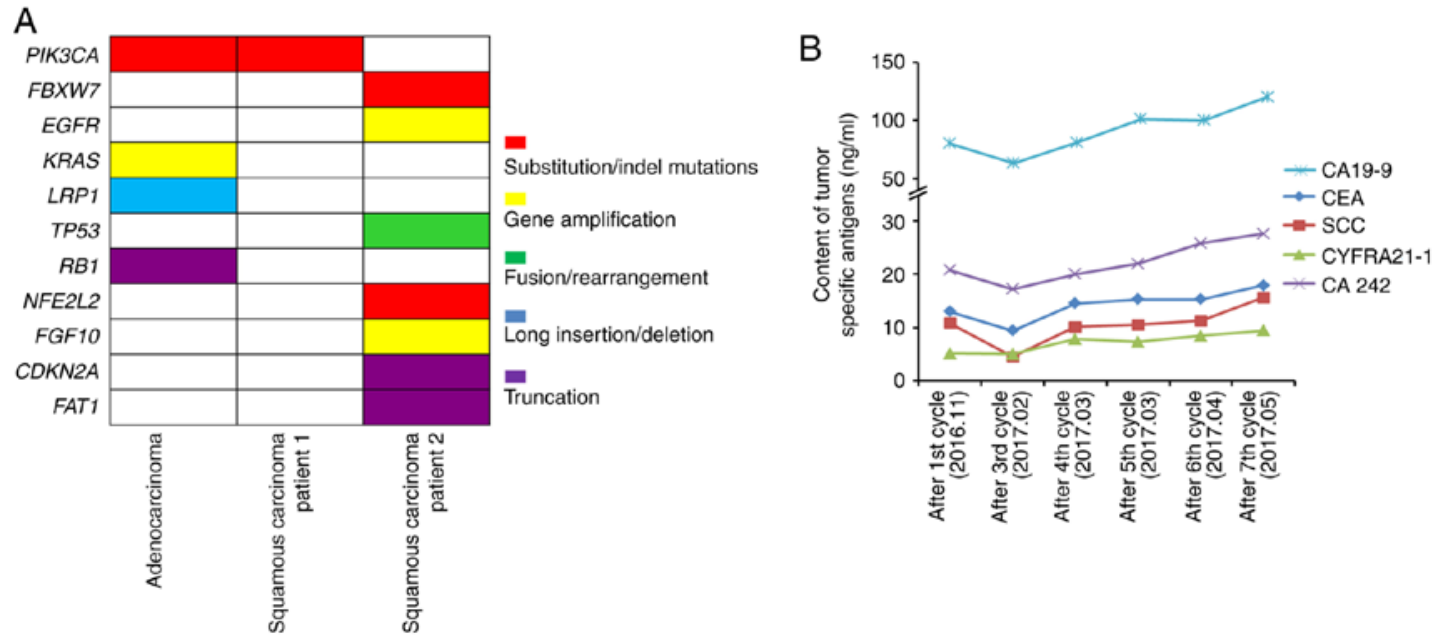

Figure 5. Characterization of genomic alterations and tumor-specific antigen levels. (A) Clinically relevant genomic alterations observed in 3 patients resistant to anti-PD-1 therapies. Red represents substitution/Indel mutations, yellow represents gene amplification mutations, green represents fusion/rearrangement mutations, blue represents long insertion/deletion mutations, and purple represents truncation mutations. (B) Tumor specific antigens of patient 3 had been monitored during the treatment with pembrolizumab. PD-1, programmed death-1; CEA, carcinoembryonic antigen; SCC, squamous cell carcinoma.

\section{Discussion}

Rapid advances in NGS technology have made it feasible to profile an increasing number of cancer-associated gene alterations using small quantities of FFPE-derived DNA. Numerous studies have been devoted to genomic variations in NSCLC. Tsoulos et al (29) collected 512 tumors from Greece for NGS analysis and identified mutations in TP53, KRAS, and EGFR to be the most common, which was consistent with other data from western population patients $(30,31)$. For Chinese NSCLC patients, it was reported that there was a higher frequency of EGFR, KRAS, and ALK mutations compared with western patients (32). The current study included an increased number of samples from Chinese NSCLC patients. Consistent with the previous study, the most frequent GAs were confirmed to be the mutations of TP53, EGFR, KRAS, CDKN2A, LRP1B and TERT (30-32). Furthermore, according to a previous study, in Chinese NSCLC patients, the mutation frequencies of EGFR (52.47 vs. 20.0\%, respectively) and ALK (8.32 vs. $4.1 \%$, respectively) were higher, and the mutation frequency of KRAS (13.36 vs. 32.0\%, respectively) was significantly lower compared with western NSCLC patients (33).

TMB was calculated by measuring the number of gene mutations in the tumor genome. TMB-H has been reported to be positively correlated with response to PD-1 inhibitors, and patients with TMB-H may benefit from immunotherapy (34). A previous study showed that there was an association between TMB and the efficacy of immunotherapy, and a significant TMB difference was found between patients who experienced partial response, stable disease and progressive disease (35). In the current study, TMB-H was associated with gender, and the TMB-H value was significantly higher in males than in females, indicating that male patients may possibly benefit from immunotherapy.

The incidence of NSCLC is higher in older patients than in younger patients (36). A study by Goodman et al (37) examined the relationship between TMB and outcome in diverse cancer treated with immunotherapy, and found that patients aged $\geq 60$ were associated with high $\mathrm{TMB}$ while those aged $<60$ were associated with low to intermediate TMB. The current study also detected an association between the TMB value and the age of NSCLC patients. Among the 28 patients aged 81-92 years, 11 (39.29\%) had TMB-H, while among the 101 patients aged 25-40 years, only $2(1.98 \%)$ patients had TMB-H. The rate of TMB-H was significantly lower in both 25-40 years-old patients and 51-60 years-old patients than that in 61-92 years old patients. These results indicated that in younger patients, although the incidence of NSCLC is lower, patients may benefit less from immunotherapy.

NSCLC is usually diagnosed at an advanced stage and surgical excision of the tumor is challenging. Wang et al (38) showed that increased TMB was significantly associated with advanced tumor stage in gynecological cancers. In the current study, a similar association between the TMB value and tumor stage was identified. A previous report showed that NSCLC patients with KRAS mutations had significantly higher rate of TMB-H (>10 mut/Mb) than patients with EGFR, ALK, ROS1 or MET mutations (39). In the current study, the most frequently mutated genes, TP53, EGFR, KRAS, CDKN2A and LRP1B, were more common among TMB-H patients than TMB-L patients, suggesting that numerous Chinese NSCLC patients, especially those with TP53, EGFR, KRAS, CDKN2A and LRP1B mutations, may benefit from immunotherapy.

Previous studies have shown that the incidence of lung cancer is at least 20-fold higher among smokers than in non-smokers $(40,41)$. It had been reported that smoking was more significantly associated with lung squamous cell carcinoma than with adenocarcinoma (42). Based on the 1,511 patients with smoking status information included in the current study, the results also showed that smoking was more prevalent among males than in females. The incidence of lung squamous cell carcinoma was high in male patients who were continuously smoking more than 10 years, indicating that the smoking status may be associated with the incidence of lung squamous cell carcinoma. Association analysis between smoking and lung squamous cell carcinoma also supported 
this conclusion. The present study found the TMB-H to be associated with the smoking status of patients, implying the potential benefit from immunotherapy on smoking patients, which is consistent with the results of a previous study (43).

PD-1 and PD-L1 can be targeted to maintain the function of effector T-cells. Monoclonal antibodies are used as checkpoint inhibitors in order to inhibit the interaction of PD-1 and PD-L1 (44). Certain characteristics, such as gender, type of tumor, mutations, translocation of genes and metastasis, determine the effectiveness of these inhibitors (45). Acquired resistance occurs in most patients after an initial response to the PD-1/PD-L1 blockade, which then leads to disease progression or relapse (46). The PI3K/Akt and mTOR signaling pathways are important to numerous physiological and pathological conditions. PI3K has been found to be overexpressed in ovarian and cervical cancer $(47,48)$. PI3K mutations have been identified in breast cancer, glioblastoma and gastric cancer (49). Zhao et al (50) reported that an anti-PD-L1 antibody influenced the expression of PTEN, PI3K and Akt, and the PD-L1 blockade inhibited the tumor growth and metastasis by modulating the PI3K/Akt/mTOR signaling pathway. A study by Deken et al (51) analyzed the effect of dual MAPK pathway inhibition in combination with $\mathrm{PI} 3 \mathrm{~K} / \mathrm{mTOR}$ pathway inhibition and its potential synergy with PD-1 blockade in a melanoma mouse model. The current study found 3 NSCLC patients harboring the mutation of PI3K/mTOR pathway-related genes who developed resistance after at least 6 cycles of anti-PD-1 immunotherapies with pembrolizumab or nivolumab, suggesting that these mutations may be related to PD-1 blockade resistance. Following treatment with mTOR inhibitors, stable disease or shrinkage of the tumor was achieved in these patients. These data showed that NSCLC with $\mathrm{PI} 3 \mathrm{~K} / \mathrm{mTOR}$ mutations was sensitive to mTOR inhibitors in these 3 patients. However, the limited number of cases is a limitation, and more cases in a larger patient population need to be studied for further validation.

In conclusion, the current findings suggested that TMB could be a potential predictive biomarker for Chinese NSCLC patients. PI3K/mTOR pathway alterations occurred more frequently in NSCLC patients with higher TMB values, and therefore, mTOR inhibitors might be a potential strategy for those who harbor mutations in the PI3K/mTOR pathway after resistance to PD-1/PD-L1 blockade therapies.

\section{Acknowledgements}

Not applicable.

\section{Funding}

No funding was received.

\section{Availability of data and materials}

The datasets used and analyzed in this study are available from the corresponding author on reasonable request.

\section{Authors' contributions}

JH, YS, TS, XP and YW collected patient consents and samples, analyzed data, and wrote the manuscript. XS, SZ,
JS, MY, AW, WS, KW and AL contributed to bioinformatics analysis, design the study and reviewed the manuscript. XP and YW designed and supervised the study. All authors read and approved the final manuscript.

\section{Ethics approval and consent to participate}

This study was approved by the Ethics Committees of The First People's Hospital of Yunnan Province, Affiliated Hospital of Hebei University, Fujian Provical Hospital and The Affiliated Hospital of Qingdao University. Informed consent for participation was obtained from all patients.

\section{Patient consent for publication}

Not applicable.

\section{Competing interests}

The authors declare that they have no competing interests.

\section{References}

1. Linnoila I: Pathology of non-small cell lung cancer. New diagnostic approaches. Hematol Oncol Clin North Am 4: 1027-1051, 1990.

2. Nemunaitis J,Papai Z, Léna H,Losonczy G, Forget F, Chouaid C, Szczesna A, Gervais R, Ottensmeier CH, Beck J, et al: TG4010 immunotherapy plus chemotherapy as first-line treatment of advanced non small cell lung cancer (NSCLC): Phase IIb results of the TIME trial. J Immunother Cancer 3 (Suppl 2): P441, 2015.

3. Rolfo C, Caglevic C, Santarpia M, Araujo A, Giovannetti E, Gallardo CD, Pauwels $\mathrm{P}$ and Mahave M: Immunotherapy in NSCLC: A promising and revolutionary weapon. Adv Exp Med Biol 995: 97-125, 2017.

4. Mahoney KM, Freeman GJ and McDermott DF: The next immune-checkpoint inhibitors: PD-1/PD-L1 blockade in melanoma. Clin Ther 37: 764-782, 2015.

5. Hamanishi J, Mandai M, Matsumura N, Abiko K, Baba T and Konishi I: PD-1/PD-L1 blockade in cancer treatment: Perspectives and issues. Int J Clin Oncol 21: 462-473, 2016.

6. Teo MY, Seier K, Ostrovnaya I, Regazzi AM Kania BE, Moran MM, Cipolla CK, Bluth MJ, Chaim J, Al-Ahmadie $\mathrm{H}$, et al: Alterations in DNA damage response and repair genes as potential marker of clinical benefit from PD-1/PD-L1 blockade in advanced urothelial cancers. J Clin Oncol 36: 1685-1694, 2018.

7. Jia L, Zhang Q and Zhang R: PD-1/PD-L1 pathway blockade works as an effective and practical therapy for cancer immunotherapy. Cancer Biol Med 15: 116-123, 2018.

8. Alexandrov LB, Nik-Zainal S, Wedge DC, Aparicio SA, Behjati S, Biankin AV, Bignell GR, Bolli N, Borg A, Børresen-Dale AL, et al: Signatures of mutational processes in human cancer. Nature 500: 415-421, 2013.

9. Cantley LC: The phosphoinositide 3-kinase pathway. Science 296: 1655-1657, 2002.

10. Shaw RJ and Cantley LC: Ras, PI(3)K and mTOR signalling controls tumour cell growth. Nature 441: 424-430, 2006.

11. Bader AG, Kang S, Zhao L and Vogt PK: Oncogenic PI3K deregulates transcription and translation. Nat Rev Cancer 5: 921-929, 2005.

12. Mao JH, Kim IJ, Wu D, Climent J, Kang HC, DelRosario R and Balmain A: FBXW7 targets mTOR for degradation and cooperates with PTEN in tumor suppression. Science 321: 1499-1502, 2008.

13. Loi S, Haibe-Kains B, Majjaj S, Lallemand F, Durbecq V, Larsimont D, Gonzalez-Angulo AM, Pusztai L, Symmans WF, Bardelli A, et al: PIK3CA mutations associated with gene signature of low mTORC1 signaling and better outcomes in estrogen receptor-positive breast cancer. Proc Natl Acad Sci USA 107: 10208-10213, 2010. 
14. Engelman JA, Luo J and Cantley LC: The evolution of phosphatidylinositol 3-kinases as regulators of growth and metabolism. Nat Rev Genet 7: 606-619, 2006.

15. Yip PY: Phosphatidylinositol 3-kinase-AKT-mammalian target of rapamycin (PI3K-Akt-mTOR) signaling pathway in non-small cell lung cancer. Transl Lung Cancer Res 4: 165-176, 2015.

16. Balsara BR, Pei J, Mitsuuchi Y, Page R, Klein-Szanto A, Wang $\mathrm{H}$, Unger $\mathrm{M}$ and Testa JR: Frequent activation of AKT in non-small cell lung carcinomas and preneoplastic bronchial lesions. Carcinogenesis 25: 2053-2059, 2004.

17. Tsurutani J, Fukuoka J, Tsurutani H, Shih JH, Hewitt SM, Travis WD, Jen J and Dennis PA: Evaluation of two phosphorylation sites improves the prognostic significance of Akt activation in non-small-cell lung cancer tumors. J Clin Oncol 24: 306-314, 2006.

18. Wang MH, Shi WW, Zhang P, Zhao SH, Cao JY, Chen MW, Shou T, Hu J, Tang XF and Song TQ: A model based adjustment for tumor mutational burden across different tumor types. Ann Oncol 28 (Suppl 7): vii13, 2017.

19. Yuza K, Nagahashi M, Watanabe S, Takabe $K$ and Wakai T: Hypermutation and microsatellite instability in gastrointestinal cancers. Oncotarget 8: 112103-112115, 2017.

20. Dong ZY, Zhong WZ, Zhang XC, Su J, Xie Z, Liu SY, Tu HY, Chen HJ, Sun YL, Zhou Q, et al: Potential predictive value of TP53 and KRAS mutation status for response to PD-1 blockade immunotherapy in lung adenocarcinoma. Clin Cancer Res 23 3012-3024, 2017

21. Ali SM, Yao M, Yao J, Wang J, Cheng Y, Schrock AB, Chirn GW, Chen H, Mu S, Gay L, et al: Comprehensive genomic profiling of different subtypes of nasopharyngeal carcinoma reveals similarities and differences to guide targeted therapy. Cancer 123: 3628-3637, 2017.

22. Cibulskis K, Lawrence MS, Carter SL, Sivachenko A, Jaffe D, Sougnez C, Gabriel S, Meyerson M, Lander ES and Getz G: Sensitive detection of somatic point mutations in impure and heterogeneous cancer samples. Nat Biotechnol 31: 213-219, 2013.

23. Ye K, Schulz MH, Long Q, Apweiler R and Ning Z: Pindel: A pattern growth approach to detect break points of large deletions and medium sized insertions from paired-end short reads. Bioinformatics 25: 2865-2871, 2009.

24. Cingolani P, Platts A, Wang le L, Coon M, Nguyen T, Wang L, Land SJ, Lu X and Ruden DM: A program for annotating and predicting the effects of single nucleotide polymorphisms, SnpEff: SNPs in the genome of Drosophila melanogaster strain w1118; iso-2; iso-3. Fly (Austin) 6: 80-92, 2012.

25. Boeva V, Popova T, Bleakley K, Chiche P, Cappo J Schleiermacher G, Janoueix-Lerosey I, Delattre O and Barillot E: Control-FREEC: A tool for assessing copy number and allelic content using next-generation sequencing data. Bioinformatics 28: 423-425, 2012.

26. Cao J, Chen L, Li H, Chen H, Yao J, Mu S, Liu W, Zhang P, Cheng Y, Liu B, et al: An accurate and comprehensive clinical sequencing assay for cancer targeted and immunotherapies. Oncologist 24: e1294-e1302, 2019.

27. Thorvaldsdóttir H, Robinson JT and Mesirov JP: Integrative genomics viewer (IGV): High-performance genomics data visualization and exploration. Brief Bioinform 14: 178-192, 2013.

28. Detterbeck FC, Boffa DJ, Kim AW and Tanoue LT: The eighth edition lung cancer stage classification. Chest 151: 193-203, 2017.

29. Tsoulos N, Papadopoulou E, Metaxa-Mariatou V, Tsaousis G Efstathiadou C, Tounta G, Scapeti A, Bourkoula E, Zarogoulidis P, Pentheroudakis G, et al: Tumor molecular profiling of NSCLC patients using next generation sequencing. Oncol Rep 38: 3419-3429, 2017.

30. Scoccianti C, Vesin A, Martel G, Olivier M, Brambilla E, Timsit JF, Tavecchio L, Brambilla C, Field JK and Hainaut P, European Early Lung Cancer Consortium: Prognostic value of TP53, KRAS and EGFR mutations in nonsmall cell lung cancer: The EUELC cohort. Eur Respir J 40: 177-184, 2012.

31. Vavalà T, Monica V, Lo Iacono M, Mele T, Busso S, Righi L, Papotti M, Scagliotti GV and Novello S: Precision medicine in age-specific non-small-cell-lung-cancer patients: Integrating biomolecular results into clinical practice-A new approach to improve personalized translational research. Lung Cancer 107: $84-90,2017$.

32. Wen S, Dai L, Wang L, Wang W, Wu D, Wang K, He Z, Wang A, Chen $\mathrm{H}$, Zhang P, et al: Genomic signature of driver genes identified by target next-generation sequencing in chinese non-small cell lung cancer. Oncologist 24: e1070-e1081, 2019.
33. Suh JH, Johnson A, Albacker L, Wang K, Chmielecki J, Frampton G, Gay L, Elvin JA, Vergilio JA, Ali S, et al: Comprehensive genomic profiling facilitates implementation of the national comprehensive cancer network guidelines for lung cancer biomarker testing and identifies patients who may benefit from enrollment in mechanism-driven clinical trials. Oncologist 21: 684-691, 2016.

34. Chalmers ZR, Connelly CF, Fabrizio D, Gay L, Ali SM, Ennis R, Schrock A, Campbell B, Shlien A, Chmielecki J, et al: Analysis of 100,000 human cancer genomes reveals the landscape of tumor mutational burden. Genome Med 9: 34, 2017.

35. Ricciuti B, Kravets S, Dahlberg SE, Umeton R, Albayrak A, Subegdjo SJ, Johnson BE, Nishino M, Sholl LM and Awad MM: Use of targeted next generation sequencing to characterize tumor mutational burden and efficacy of immune checkpoint inhibition in small cell lung cancer. J Immunother Cancer 7: 87, 2019.

36. Fan H, Shao ZY, Xiao YY, Xie ZH, Chen W, Xie H, Qin GY and Zhao NQ: Incidence and survival of non-small cell lung cancer in Shanghai: A population-based cohort study. BMJ Open 5: e009419, 2015.

37. Goodman AM, Kato S, Bazhenova L, Patel SP, Frampton GM, Miller V, Stephens PJ, Daniels GA and Kurzrock R: Tumor mutational burden as an independent predictor of response to immunotherapy in diverse cancers. Mol Cancer Ther 16: 2598-2608, 2017.

38. Wang M, Fan W, Ye M, Tian C, Zhao L, Wang J, Han W, Yang W, $\mathrm{Gu} \mathrm{C}, \mathrm{Li} \mathrm{M}$, et al: Molecular profiles and tumor mutational burden analysis in Chinese patients with gynecologic cancers. Sci Rep 8: 8990, 2018.

39. Berland L, Heeke S, Humbert O, Macocco A, Long-Mira E, Lassalle S, Lespinet-Fabre V, Lalvée S, Bordone O, Cohen C, et al: Current views on tumor mutational burden in patients with non-small cell lung cancer treated by immune checkpoint inhibitors. J Thorac Dis 11 (Suppl 1): S71-S80, 2019.

40. Sasco AJ, Secretan MB and Straif K: Tobacco smoking and cancer: A brief review of recent epidemiological evidence. Lung Cancer 45 (Suppl 2): S3-S9, 2004.

41. Lubin JH, Alavanja MC, Caporaso N, Brown LM, Brownson RC, Field RW, Garcia-Closas M, Hartge P, Hauptmann M, Hayes RB, et al: Cigarette smoking and cancer risk: Modeling total exposure and intensity. Am J Epidemiol 166: 479-489, 2007.

42. Egawa H, Furukawa K, Preston D, Funamoto S, Yonehara S, Matsuo T, Tokuoka S, Suyama A, Ozasa K, Kodama K and Mabuchi K: Radiation and smoking effects on lung cancer incidence by histological types among atomic bomb survivors. Radiat Res 178: 191-201, 2012.

43. Nagahashi M, Sato S, Yuza K, Shimada Y, Ichikawa H, Watanabe S, Takada K, Okamoto T, Okuda S, Lyle S, et al: Common driver mutations and smoking history affect tumor mutation burden in lung adenocarcinoma. J Surg Res 230: 181-185, 2018

44. Alsaab HO, Sau S, Alzhrani R, Tatiparti K, Bhise K, Kashaw SK and Iyer AK: PD-1 and PD-L1 checkpoint signaling inhibition for cancer immunotherapy: Mechanism, combinations, and clinical outcome. Front Pharmacol 8: 561, 2017.

45. D'Incecco A, Andreozzi M, Ludovini V, Rossi E, Capodanno A, Landi L, Tibaldi C, Minuti G, Salvini J, Coppi E, et al: PD-1 and PD-L1 expression in molecularly selected non-small-cell lung cancer patients. Br J Cancer 112: 95-102, 2015.

46. Bai J, Gao Z, Li X, Dong L, Han W and Nie J: Regulation of PD-1/PD-L1 pathway and resistance to PD-1/PD-L1 blockade. Oncotarget 8: 110693-110707, 2017.

47. Shayesteh L, Lu Y, Kuo WL, Baldocchi R, Godfrey T, Collins C, Pinkel D, Powell B, Mills GB and Gray JW: PIK3CA is implicated as an oncogene in ovarian cancer. Nat Genet 21: 99-102, 1999.

48. Ma YY, Wei SJ, Lin YC, Lung JC, Chang TC, Whang-Peng J, Liu JM, Yang DM, Yang WK and Shen CY: PIK3CA as an oncogene in cervical cancer. Oncogene 19: 2739-2744, 2000.

49. Samuels Y, Wang Z, Bardelli A, Silliman N, Ptak J, Szabo S, Yan H, Gazdar A, Powell SM, Riggins GJ, et al: High frequency of mutations of the PIK3CA gene in human cancers. Science 304: 554,2004

50. Zhao L, Li C, Liu F, Zhao Y, Liu J, Hua Y, Liu J, Huang J and Ge C: A blockade of PD-L1 produced antitumor and antimetastatic effects in an orthotopic mouse pancreatic cancer model via the PI3K/Akt/mTOR signaling pathway. Onco Targets Ther 10: 2115-2126, 2017.

51. Deken MA, Gadiot J, Jordanova ES, Lacroix R, van Gool M, Kroon P, Pineda C, Geukes Foppen MH, Scolyer R, Song JY, et al: Targeting the MAPK and PI3K pathways in combination with PDI blockade in melanoma. Oncoimmunology 5: e1238557, 2016. 